\title{
Cardioversor-Desfibrilador na Prevenção Primária de Morte Súbita: para Todos ou para Poucos?
}

\author{
Cardioverter-Defibrillator in the Primary Prevention of Sudden Death: for All or for a Few? \\ Leandro loschpe Zimerman \\ Hospital de Clínicas de Porto Alegre e Hospital São Francisco-ISCMPA, Porto Alegre, RS - Brasil
}

Os cardioversores-desfibriladores implantáveis (CDI) têm sido usados há mais de duas décadas para prevenção de morte súbita. Inicialmente recomendados para a prevenção secundária, atualmente têm na prevenção primária a sua indicação mais comum. Registro norte-americano recente mostrou que dos 108.341 implantes realizados em 2006, $85.823(79,2 \%)$ foram para prevenção primária e somente $22.518(20,8 \%)$ para secundária1. Vários ensaios clínicos têm procurado definir quais são os pacientes que merecem essa forma de tratamento. O mais recente e maior destes é o Sudden Cardiac Death in Heart Failure (SCD-Heft) ${ }^{2}$ que seguiu 2.521 pacientes com ICC classe funcional II-III e FE $\leq 35 \%$ em três grupos (placebo, amiodarona e implante de CDI) por um tempo médio de 45,5 meses. Neste, observou-se uma diminuição em mortalidade total no grupo com CDI, com $23 \%$ de redução de risco relativo no grupo CDI, e ausência de benefício com o uso da amiodarona.

Como esses ensaios clínicos foram realizados basicamente em países desenvolvidos, em uma realidade diferente da brasileira, a aplicabilidade desses dados é freqüentemente questionada. No presente artigo $^{3}$, buscou-se validar os resultados para a população brasileira ao se compararem os pacientes do SCD-Heft àqueles que apresentassem critérios de inclusão do SCD-Heft e estivessem de um ambulatório de insuficiência cardíaca de um hospital do nosso meio. A comparação entre os grupos mostrou pequenas diferenças: o percentual de pacientes isquêmicos é ligeiramente maior (63\% vs. $53 \%, p=0,05)$ e o uso de digoxina é menor nos pacientes do presente estudo (35\% vs. $70 \%$; $p<0,001)$. Em relação à digoxina, não se espera alteração em mortalidade com seu uso, o que torna este possível fator de confusão menor importante. O percentual ligeiramente superior de isquêmicos poderia ter significado em termos de mortalidade, já que estes costumam apresentar taxas maiores que os não-isquêmicos, mas isso não foi observado. Os dados mais importantes do ponto de vista de alteração de mortalidade, como a fração de ejeção e o uso de betabloqueadores e inibidores da ECA, são similares entre os dois trabalhos. No SCD-Heft, o benefício observado do

\section{Palavras-chave}

Desfibriladores implantáveis, prevenção primária, morte súbita, custos de cuidados de saúde

Correspondência: Leandro loschpe Zimerman •

Avenida Iguassu, 176/402 - Petrópolis 90470-430 - Porto Alegre - RS - Brasil

E-mail: zimerman@cardiol.br, Izimerman@terra.com.br

Artigo recebido em 21/05/08; revisado em 21/05/08;

aceito em 21/05/08. implante de CDI ocorreu exclusivamente em pacientes com classe funcional II da NYHA, que representavam 70\% do total. Esse percentual foi de $67 \%$ no presente estudo, reafirmando a similaridade entre as populações. A conclusão a que se chega é que pacientes acompanhados em ambulatório de ICC em nosso meio são similares aos randomizados no SCD-Heft, indicando que os resultados deste ensaio clínico podem ser aplicados em nosso meio.

No entanto, outro ponto muito discutido em relação ao implante de CDI em nosso meio é o custo-efetividade. Os autores do presente trabalho sugerem que essa terapêutica não é usada mais regularmente em razão do impacto econômico que geraria em nosso sistema de saúde. Essa discussão não é recente e ocorre também em países desenvolvidos ${ }^{4-6}$. Vários ensaios clínicos têm medido e comparado os custos do implante do CDI com outras formas de tratamento. A diferença de custos para o implante varia de U\$ 19.000, no SCD-Heft ${ }^{7}$, a U\$ 39.200, no MADIT II $^{8}$. O custo da terapia deve ser julgado em conjunto com o benefício que ela apresenta. Do mesmo modo que o custo, o NNT, número necessário de pacientes tratados para evitar um desfecho, é bastante variado. No MADIT II, o NNT é de 11 para 3 anos, no SCD-Heft é de 14 para 5 anos; e é de 3 para 5 anos no MUSTT e de 4 para 2,4 anos no MADIT ${ }^{9-11}$. Outro dado a ser incorporado nesse cálculo é que o custo-efetividade deve ser ajustado à tolerância do paciente ao tratamento, ou seja, ajustado à qualidade de vida. E, novamente, os dados de aumento de custo-efetividade ajustados à qualidade de vida por ano (QALY) são muito diferentes entre os diversos estudos. Considerando-se uma eficácia média, os valores de QALY encontrados são, em dólares, 34.000, 34.900, 54.100 e 70.200, para os estudos MUSTT, MADIT, MADIT II e SCDHeft, respectivamente ${ }^{12}$. Números tão diferentes geram posicionamentos diferentes. Enquanto vários autores sugerem que o implante de CDI é muito caro para ser sustentado pelo sistema de saúde público, outros não concordam com esse argumento. Camm e cols. ${ }^{13}$ consideram que o aumento do gasto para o sistema da saúde europeu seria pouco relevante, mesmo que os implantes aumentassem três vezes. Além disso, esses autores insistem que o custo anual dos CDI na Europa (0,49 bilhão de euros) é desprezível quando comparado a outras terapêuticas correntes, como a cirurgia de revascularização miocárdica (2,12 bilhões) ou angioplastia coronária (2,7 bilhões), ou com outros custos, como a ineficiência na administração hospitalar (64 bilhões). Talvez o dado mais consistente ao se analisarem os vários trabalhos seja o de que quanto maior a estratificação de risco, melhores são o NNT e o custo-efetividade. No entanto, o principal risco relacionado ao uso de métodos de estratificação 


\section{Editorial}

mais específicos é a possibilidade de haver diminuição da sensibilidade, reduzindo assim o número de pessoas que poderiam se beneficiar.

Dados brasileiros que avaliam o custo-efetividade do implante de cardioversores-desfibriladores no setor público para prevenção primária estão sendo estudados, mas ainda não publicados*. Um estudo realizado no Hospital de Clínicas de Porto Alegre (RS) mostrou uma diferença de custoefetividade do $\mathrm{CDI}$ em relação ao tratamento convencional, em reais, de 72.280/QALY. Se o preço de implante e a troca de gerador do CDI sofressem uma redução de $50 \%$, essa relação seria de 38.436/QALY. E se os pacientes selecionados fossem somente os de mais alto risco, como os do MADIT, esse valor seria de 59.738/QALY.

O presente trabalho traz uma contribuição importante ao mostrar que os dados de um grande ensaio clínico podem ser aplicados à nossa população. Os cardioversores-desfibriladores são úteis na redução de mortalidade. O grande desafio, em nosso país e em todo o mundo, é encontrar métodos mais precisos de estratificação, associados a CDI mais baratos e de durabilidade cada vez maior, para aumentar o número de beneficiados com um bom custo-efetividade.

* Comunicação pessoal

\section{Referências}

1. Hammill SC, Stevenson LW, Kadish AH, Kremers MS, Heidenreich P, Lindsay $\mathrm{BD}$, et al. Review of the registry's first year, data collected, and future plans. Heart Rhythm. 2007; 4: 1260-3.

2. Bardy G, Lee K, Mark D, Poole JE, Packer DL, Boineau R, et al. Amiodarone or an implantable cardioverter-defibrillator for congestive heart failure (SCDHeft) Trial. N Eng J Med. 2005; 352: 225-37

3. Hadid C, Avellana P, Di Toro D, Fernández Gomez C, Visser M, Prieto N. Long-term follow-up of patients with indication for a implantable defibrillator for primary prevention of death. Arq Bras Cardiol. 2008; 90 (5): 311-5.

4. Zipes D. Implantable cardioverter-defibrillator: a Wolkswagen or a Rolls Royce: how much will we pay to safe a Life? Circulation. 2001; 103: 1372-4.

5. Hlatky M, Mark D. The high cost of implantable defibrillators. Eur Heart 2007; 28: 388-91.

6. Heidelbüchel $\mathrm{H}$. The cost of the implantable defibrillators: how the perception of reality depends on perspective. Eur Heart J. 2007; 28: 386-7.

7. Mark D, Nelson C, Anstrom K, Al-Khatib SM, Tsiatis AA, Cowper PA, et al. Cost-effectiveness of defibrillator therapy or amiodarone in chronic stable heart failure: results from the Sudden Cardiac Death in Heart Failure Trial
(SCD-Heft). Circulation. 2006; 114: 135-42.

8. Zwanziger J, Hall J, Dick A, Zhao H, Mushlin Al, Hahn RM, et al. The cost effectiveness of implantable cardioverter-defibrillators: results from the Multicenter Automatic Defibrillator Implantation Trial (MADIT) -II. J Am Coll Cardiol. 2006; 47: 2310-8.

9. Moss A, Zareba W, Hall J, Wilber DJ, Cannom DS, Klein H, et al. Prophylactic implantation of a defibrillator in patients with myocardial infarction and reduced ejection fraction. N Eng J Med. 2002; 346: 877-83.

10. Moss A, Hall J, Cannom D, Daubert JP, Higgins SL, Klein H, et al. Improved survival with an implantable defibrillator in patients with coronary disease at high risk of ventricular arrhythmias. N Eng J Med. 1996; 335: 1933-40.

11. Buxton A, Lee K, Fisher J, Josephson ME, Prystowsky EN, Hafley G. A randomized study for the prevention of sudden death in patients with coronary artery disease. N Eng J Med. 1999; 341: 1882-90.

12. Sanders G, Hlatky M, Owens D. Cost-effectiveness of implantable cardioverter-defibrillators. N Eng J Med. 2005; 353: 1471-80.

13. Camm J, Klein H, Nisan S. The cost of implantable defibrillators: perceptions and reality. Eur Heart J. 2007; 28: 392-7. 